\title{
Dual Diagnosis Enhanced Programs
}

\author{
Kenneth Minkoff, MD
}

In 2001, the American Society of Addiction Medicine Patient Placement Criteria, Second Edition, Revised (ASAM PPC-2R) (American Society of Addiction Medicine, 2001) introduced the concepts of dual diagnosis capability (DDC) and dual diagnosis enhanced (DDE) addiction programs into the national lexicon. The original definitions of DDC and DDE addiction programs were relatively brief.

DDC programs "address co-occurring . . . disorders in their policies and procedures, assessment, treatment planning, program content, and discharge planning" (American Society of Addiction Medicine, 2001, p. 362; Center for Substance Abuse Treatment, 2005, p. 33) so that program staff are able to address co-occurring disorders routinely in relapse skills, recovery environment, and readiness to change "through individual and group program content."

DDE programs are able to provide primary substance abuse treatment to clients who are, as compared to those routinely treated in DDC programs, "more symptomatic and/or functionally impaired as a result of their cooccurring mental disorder" (American Society of Addiction Medicine, 2001, p. 10, Center for Substance Abuse Treatment, p. 33).

Nonetheless, the fundamental message was very important: co-occurring disorder is an expectation in addiction treatment settings, and a continuum of addiction programs needs to incorporate routine DDC into its full array of services and plan for some DDE service components in order to provide access to episodes of addiction treatment for individuals who would be unable to receive treatment routinely in DDC programs. Further, fewer and

Kenneth Minkoff, MD, is Clinical Assistant Professor of Psychiatry, Harvard Medical School, Boston, MA.

Address correspondence to: Kenneth Minkoff, MD, 100 Powdermill Road \# 319, Acton, MA 01720 (E-mail: kminkov@aol.com).

Journal of Dual Diagnosis, Vol. 4(3), 2008

Available online at http://jdd.haworthpress.com 
fewer programs would be able to maintain themselves as "addiction only" over time.

In the years since the release of the ASAM PPC-2R, the application of the concept of DDC has become much more widespread and much more clearly articulated. DDC as a concept is now routinely applied to mental health programs (and both adult and child services), and previous issues of this column have discussed the criteria for DDC implementation at great length (Minkoff \& Cline, 2006). Further, state and county systems are working to develop infrastructures that would support all programs developing DDC at minimum as a core expectation (Minkoff \& Cline, 2004; 2005). Tools have been developed to allow programs to self-assess for DDC (Minkoff \& Cline, 2001) or to be assessed for DDC (McGovern, Matzkin, \& Giard, 2007). Several state and county systems have developed system-specific regulations, program audit tools, and internal processes of monitoring and technical assistance to support DDC development.

As more systems develop DDC as a universal feature, there is increasing interest in "taking the next step" and starting to define program standards for DDE programs, with the understanding that within a comprehensive continuous integrated system of care (Minkoff \& Cline, 2004), system design should incorporate a small selection of DDE programs along with universal attainment of DDC. Some systems have hypothesized that since DDC programs can be created within base funding and base staffing, DDE programs need to be defined by either additional funding; additional, more highly trained staff; or both.

Although at first glance it makes sense for systems to want to create "enhanced" programming, there is a significant problem with moving in this direction, namely, that as yet there is no clear conceptualization of what DDE actually means, beyond the initial description in the ASAM PPC-2R, which not only is quite brief but only applies to addiction programs. Further, simply adding funding or adding more expensive staffing may not produce good value without a clear model for program design that matches the needs of the population being served and that is properly positioned within the design of the larger system of care.

For this reason, it may be timely to dedicate this column to a broader discussion of the meaning of DDE.

Let's begin with a bit of history. The precursor to the concepts of DDC and DDE dates back to a series of articles and publications during the 1990s (Minkoff, 1991; 1998). The description of basic and specialized dual diagnosis programs throughout the system that was a key feature of the 1998 report informed a consensus project in Massachusetts (Barreira, 
Espey, Fishbein, Moran, \& Flannery, 2000) that produced a system design template (Minkoff, 1999) that included a description of an array of what would now be termed DDC and DDE programs. This work presaged and informed the development of the ASAM PPC-2R (Minkoff, Zweben, Rosenthal, \& Ries, 2003).

These original conceptualizations allow us to understand more clearly the nature of DDE programs. First and most important, there was originally a clear distinction between DDE mental health $(\mathrm{MH})$ programs and DDE chemical dependency $(\mathrm{CD})$ programs. This will be described in more detail in the next few paragraphs.

DDE-CD programs, as described in the ASAM PPC-2R, were intended to characterize addiction treatment programs (or tracks in programs), at any level of care, that are designed to provide addiction treatment to a full cohort of clients with co-occurring disorders and moderate to severe active psychiatric symptomatology or baseline psychiatric disability. Examples of such clients would be individuals seeking addiction treatment who also had active post-traumatic stress disorder symptoms with significant flashbacks and nightmares or individuals seeking addiction treatment who also had baseline schizophrenia with moderate impairment. In general, such individuals as a group require a higher staffing ratio than DDC programs (which routinely work with individuals with co-occurring disorders with only mild to moderate impairment) as well as greater on-site access to staff with MH expertise, more program modifications, smaller groups, and so on. DDC programs can often accommodate an occasional individual client with a higher level of need, but cannot manage a full cohort of such clients without the full range of accommodations mentioned above. Note that simply adding more staff alone or hiring more highly trained staff alone does not result in a DDE-CD program. What is critical is that the program itself is designed at every level to match the functional needs of a more impaired population in an addiction treatment environment.

In short, DDE-CD programs have a higher resource requirement than DDC-CD programs and a slightly different staffing mix, but the program content (e.g., addiction treatment groups, MH symptom management, and skill building) is fairly similar. In addition, just as in a DDC-CD program, all staff still need to have core competency working with co-occurring individuals; however, with a higher staff ratio there will be better ability to assist individuals who need more structure or support to learn how to manage their addiction effectively.

DDE-MH programs, by contrast, were originally conceptualized quite differently. These were program models that were for the most part intended 
to enhance the addiction treatment content of a standard MH program (of any type) within existing resources. The clearest example to illustrate this concept would be a DDE inpatient psychiatric unit, in which the programming on the unit provides reasonably full addiction content at the same cost as a standard DDC psychiatric inpatient unit (which would be working with patients who may be less willing or able to engage in addiction-related programming while in acute care) (Minkoff, 1989; Ries, et al., 2001). In the same manner, one could contrast a DDE partial hospital program, day treatment program, group residential program, and so on with its DDC counterpart based on the intensity of the addiction content in the program and the extent to which the program is specifically focused on providing such content to individuals with co-occurring disorders. Again, note that simply hiring more staff with an addiction background does not create a DDE-MH program; the critical feature is redesigning the program content (policies, procedures, manuals, groups, etc.) and function to have a greater focus on integrating specific attention to addiction treatment needs within an $\mathrm{MH}$ setting. All staff still need to have fundamental co-occurring disorder competencies (just as in a DDC-MH program), but they will be operating within a program that has different content.

Although the line between DDC and DDE-MH programs is relatively clear in programs with highly structured program content, the distinction becomes more blurred in looking at outpatient or case management programs. For example, a DDC assertive community treatment (ACT) team will be routinely working with a high percentage of individuals with cooccurring disorders as well as individuals without co-occurring disorders and will routinely need to meet some of the fidelity criteria for integrated dual disorder treatment (IDDT) teams in order to meet fidelity criteria for ACT. A full-fledged IDDT team, however, which could be considered a DDE program, is very similar to a DDC ACT program except that it concentrates exclusively on individuals with co-occurring disorders and meets more of the IDDT fidelity criteria (Drake \& Burnette, 2001) In this regard, the distinction is more one of degree than of fundamental program design.

The lesson to be learned from this discussion is primarily that the concept of DDE is fairly complex, and the definition of DDE will vary significantly depending on the type of program being discussed. Therefore, it is much more difficult (if not impossible) to establish a single set of DDE criteria than it is to establish a set of DDC criteria, either in developing an assessment tool for program capability (e.g., the Comorbidity Program Audit and Self-Survey for Behavioral Health Services [Minkoff \& Cline, 2001] 
and the Dual Diagnosis Capability in Addiction Treatment [McGovern et al., 2007]) or in developing program standards within a system of care. For this reason, it is inaccurate to conceptualize or measure DDE simply along a linear continuum on which DDC is the halfway mark. Rather, progress toward DDC can occur in any program by increasing progress in organizing core policies, procedures, and practices (Minkoff \& Cline, 2006), while measurement of DDE standards needs to look at qualitatively different program features.

In conclusion, therefore, this column is intended to be an introduction to a deeper understanding of the concept of DDE programs. As more and more systems make progress toward universal co-occurring disorder or DDC, there will be increasing interest in further delineating the characteristics not just of DDE programs generally but the specific characteristics of different types of DDE programs at different levels of care in both substance abuse settings and MH settings. As these characteristics are more clearly described and studied for each program type, standardized program assessments and program criteria for each type of DDE program can be more accurately developed.

\section{REFERENCES}

American Society of Addiction Medicine. (2001). Patient Placement Criteria (2nd ed.). Washington, DC: Author.

Barreira, P., Espey, E., Fishbein, R., Moran, D., \& Flannery, Jr., R. B. (2000). Linking substance abuse and serious mental illness service delivery systems: Initiating a statewide collaborative. Journal of Behavioral Health Services Research, 27, 107-113.

Center for Substance Abuse Treatment. (2005). Substance abuse treatment for individuals with co-occurring disorders. Treatment improvement protocol 42. Washington, DC: Author.

Drake, R. E.,\& Burnette, M. (Eds.). (2001). Integrated Dual Disorders Treatment Toolkit. Rockville, MD: Substance Abuse \& Mental Health Services Administration.

McGovern, M., Matzkin, A. L., \& Giard, J. (2007). Assessing the dual diagnosis capability of addiction treatment programs: The Dual Diagnosis Capability in Addiction Treatment (DDCAT) Index. Journal of Dual Diagnosis, 3, 111-124.

Minkoff, K. (1989). Development of an integrated model for the treatment of patients with dual diagnosis of psychosis and addiction. Hospital and Community Psychiatry, 40(10), 1031-1036.

Minkoff, K. (1991). Program components of a comprehensive integrated care system for serious mentally ill patients with substance disorders. In: K. Minkoff K \& R. E. Drake (Eds.), Dual diagnosis of major mental illness and substance disorder. New directions for mental health services, No. 50 (pp. 13-27). San Francisco, CA: Jossey-Bass. 
Minkoff, K., \& CMHS Managed Care Initiative Panel on Co-Occurring Disorders. (1998). Co-occurring psychiatric and substance disorders in managed care systems: Standards of care, practice guidelines, workforce competencies, and training curricula. Philadelphia, PA: Center for Mental Health Policy and Services Research.

Minkoff, K. (1999). Model for the desired array of services and clinical competencies for a comprehensive, continuous, integrated system of care. Worcester, MA: Center for Mental Health Services Research, University of Massachusetts Department of Psychiatry.

Minkoff, K., \& Cline, C. (2001). COMPASS (Version 1): Co-morbidity Program Audit and Self-Survey for Behavioral Health Services/Adult and Adolescent Program Audit Tool for Dual Diagnosis Capability. Albuquerque, NM: Zialogic.

Minkoff, K., \& Cline, C. (2004). Changing the world: The design and implementation of comprehensive continuous integrated systems of care for individuals with co-occurring disorders. Psychiatric Clinics of North America, 27, 727-743.

Minkoff, K., \& Cline, C. (2006). Developing welcoming systems for individuals with cooccurring disorders: The role of the comprehensive continuous integrated system of care model. Journal of Dual Diagnosis, 1, 63-89.

Minkoff, K., \& Cline, C. (2006). Dual diagnosis capability: Moving from concept to implementation. Journal of Dual Diagnosis, 2(2), 121-134.

Minkoff, K., Zweben, J., Rosenthal, R., \& Ries, R. K. (2003). Developing service intensity criteria and program categories for individuals with co-occurring disorders. Journal of Addictive Diseases, 12(suppl 1), 113-129.

Ries, R. K., Russo, J., Wingerson, D., Snowden, M., Comtois, K. A., Srebnik, D., \& RoyByrne, P. (2001). Shorter hospital stays and more rapid improvement among patients with schizophrenia and substance disorders. Psychiatric Services, 52(2), 210-214. 